\title{
PLANTAS UTILIZADAS EN TRASTORNOS GINECOLÓGICOS, PARTO Y CONTROL DE NATALIDAD EN MUJERES DE LA PARTE BAJA DEL RIO UCAYALI- AMAZONAS PERUANA
}

\author{
Lars Peter Kvist * \\ Isabel Consuelo Oré Balbín ** \\ Dedy Consuelo Llapapasca Samaniego ${ }^{* * *}$
}

\section{RESUMEN}

En las comunidades ribereñas a lo largo del Bajo Ucayali, siete varones y seis mujeres informantes fueron interrogados en forma independiente durante dos días, sobre sus conocimientos en relación a plantas medicinales con el objetivo de identificar las plantas de uso principal en afecciones femeninas y comparar este conocimiento entre varones y mujeres. Las entrevistas fueron estructuradas y dirigidas aplicando cuestionarios estandarizados.

Se presentan plantas utilizadas para solucionar problemas femeninos como trastornos menstruales, control de natalidad, trabajo de parto y algunas afecciones ginecológicas.

Las principales conclusiones son:

1) Aproximadamente 70 diferentes plantas se reportaron como útiles para esos propósitos; la mayor parte solamente se menciona una vez para el mismo propósito y algunas se reportaron repetidamente.

2) Aproximadamente $60 \%$ de los usos reportados a afecciones femeninas se refiere a plantas encontradas en huertos, chacras y purmas y $40 \%$ a especies del bosque.

\footnotetext{
* Royal Veterinary and agricultural University, United of Foresty, Thorvalbsensvej 57, DK1871 Frederiksberg C.( Copenhagen), Denmark.

** $\quad$ Morona 813, Iquitos, Perú.

*** Urbanización Río Mar, Mz. $<<\mathrm{J}>>$, Lote 18, Iquitos, Perú.
} 
Esto corresponde a los que informaron sobre todo tipo de plantas para medicina, pero si miramos solamente a las plantas para control de fertilidad y natalidad más del $75 \%$ provienen de los alrededores de los caseríos.

3) El conocimiento de hombres y mujeres es relativamente uniforme, probablemente a causa de tratamientos combinando el uso de hierbas cultivadas (más conocidas por mujeres) y árboles del bosque (más conocidos por hombres). De las hierbas cultivadas se usaron mayormente las hojas y de los árboles del bosque corteza, látex y res ma.

Palabras clave:

Perú, Río Ucayali, Plantas medicinales, Trastornos ginecológicos, Fertilidad, Natalidad, Consenso de informantes.

\begin{abstract}
ABSTRÁCT
Seven males and six females were questioned regarding medicinal plant use in communities along the lower Ucayali river of Peru. Over a two day period, utilising a formal interview and a standardised questionnaire, each of the informants searched and selected medicinal plants and described their uses. Main objectives were to identify the plants applied for women's afflictions and to compare the knowledge of men and women. Women's afflictions are here defined as problem and complications related to menstruation, fertility regulation, birth and women's abdominal diseases. The main conclusions are:

1) Nearly 70 different plants were reported as useful for women's afflictions. Two plants were reported to be useful for the same purposes by a consensus of as many as eight informants. However, the majority of the usage reports were the result of a single report.

2) Approx. 60 percent of the reports of plants described as useful for women's afflictions refer to plants found in gardens, fields and young fallow vegetation, the remaining 40 percent are forest species. This corresponds to the general distribution of all reported medicinal plants in these communities. Further more than 75 percent of the use reports citing plants used in birth control and childbirth refer to plants found near villages.

3) The knowledge of men and women is relatively uniform possibly because treatments often combine the use of cultivated herbs best known by women) with the use of forest trees (best known by men). From the cultivated herbs are mostly used leaves and from the forest trees mostly cortex, latex and resins.
\end{abstract}




\section{INTRODUCCION}

La mayoría de los trabajos etnobotánicos, en la Amazonía, se han realizado con informantes varones; raras veces se ha trabajado con informantes mujeres; corno consecuencia, existe poca información acerca del conocimiento que, sobre las plantas, tienen las mujeres; tampoco existen estudios comparativos del conocimiento etnobotánico de ambos sexos al respecto, muchos investigadores están proponiendo recientemente dichos estudios (Phillips \& Gentry, 1993).

Diversos trabajos dan cuenta del uso de los recursos del bosque en la zona de Jenaro Herrera (Chibnik \& Jong 1992; Kahn 1 988; Kahn \& Mejía 1990; Mejía 1988; Padoch \& Jong 1989; 1990; 1991; Parodi 1988; Parodi \& Freitas 1990; Peters \& Harnmond 1990), sin embargo, la información sobre el conocimiento etnornédico es escasa. Rivas (1994) demostró que muchas mujeres, en el área del proyecto, conocen y utilizan plantas con propiedades anticonceptivas. Hern (1994) aporta información sobre plantas anticonceptivas utilizadas por los indígenas Shipibo que viven en el alto río Ucayali, y Tournon (1984) también presenta datos sobre plantas medicinales utilizadas por esta etnia.

El presente artículo aporta información sobre las plantas utilizadas en problemas y enfermedades ginecológicas de las mujeres por la población ribereña en el bajo río Ucayali.

Estas plantas se presentan en cuatro principales categorías de uso: relacionadas con la menstruación, regulación de fertilidad, partos y problemas de vientre. Se discute la preparación y aplicación de las plantas y el lugar donde se encuentran, y se compara el conocimiento de los informantes femeninos y masculinos.

Este estudio es parte de un proyecto más amplio de investigación, sobre bosques en zonas inundables en el bajo Ucayali. El proyecto combina estudios botánicos, ecológicos y silviculturales en parcelas permanentes; y estudios socioeconómicos y etnobotánicos en comunidades ubicadas en la zona inundable.

\section{AREA DE ESTUDIO}

El área de estudio comprende básicamente el distrito de Jenaro Herrera, aproximadamente, a $160 \mathrm{~km}$ al sur de la ciudad de Iquitos.

Las encuestas se realizaron a lo largo de $35 \mathrm{~km}$ del bajo no Ucayali, en las comunidades ubicadas entre los caseríos de Iricahua y Santa Rosa, $15 \mathrm{~km}$ río abajo y 20 $\mathrm{km}$ río arriba de Jenaro Herrera, respectivamente (Anexo 1). La población en cada 
una de estas comunidades es de 100 (Iricahua) a 500 habitantes (Yanallpa). Jenaro Herrera es el mayor centro poblado del lugar con 4000 habitantes.

La población predominante desciende de etnias nativas locales (Cocamas, Cocamillas), particularmente en dos de los 14 caseríos que se encuentran dentro del ámbito del proyecto; mientras que un porcentaje considerable de la población ha migrado de otras localidades de la Amazonía Peruana y como tal tienen un conocimiento profundo de su ambiente local (Padoch, 1989).

Esta población, conocida localmente como «ribereños», habla español y frecuentemente se mudan de una comunidad a otra, dentro de la misma zona (Padoch, 1990).

\section{METODOLOGIA}

Para registrar la información etnobotánica se utilizó un formulario para cada planta (Anexo 2), desarrollado sobre la base de las entrevistas con un informante masculino en diciembre de 1994.

Durante marzo y abril de 1995, se entrevistó a 13 informantes; 7 hombres y 6 mujeres (Tabla 1). El tiempo utilizado para cada informante fue de dos días, pernoctando en la casa de ellos. Durante estos dos días, cada informante indicó las plantas medicinales más importantes y explicaron sus usos respectivos. Cada informante fue entrevistado en forma independiente, completándose cada vez, un formulario (Anexo 2) con los usos medicinales de una planta. Para obtener las muestras de referencia y/o para verificar la identidad de cada una de las especies registradas se ubicaron las plantas en los alrededores de sus casas, en las chacras, purinas y en el bosque.

Se colectaron muestras botánicas de la mayoría de las plantas medicinales. Duplicados de todas las colecciones han sido depositados en los herbarios AAU de Dinamarca y AMAZ de la Universidad de la Amazonía Peruana, y en el herbario de campo del Centro de Investigaciones de Jenaro Herrera.

El uso de un número considerable de plantas fue explicado sin material para documentar su identidad. Esto fue debido a que muchas especies tradicionales corresponden a especies científicas bien definidas; en otros casos no se encontraron las plantas medicinales valorizadas y en otros porque, según los informantes, no se encuentran cerca del caserío. 
Consecuentemente, se registró información sobre especies tradicionales, aunque la mayoría de ellas corresponde a especies científicas bien definidas; sin embargo, algunas especies tradicionales corresponden a varias especies de un mismo genero (por ej. cordoncillo: Piper spp.), en otros casos a varios géneros de una misma familia (por ej. suelda con suelda: Loranthaceae) o aun a diferentes géneros de diferentes familias (por ej. uña de gato: Machaerium y Uncaria). En este último caso tratamos los dos géneros como especies tradicionales diferentes. Se distinguió Machaerium y Uncaria al compararlos, y cuando faltó material se pidió a los informantes distinguir caracteres haciendo posible concluir qué género tenían en mente, es decir, hojas compuestas y alternas y látex rojizo en Machaeriurn, en contraste a hojas simples y opuestas y sin látex en Uncaria.

El número máximo de usos medicinales atribuidos a cualquier planta medicinal por un informante, fue cinco, correspondiendo este número a los cinco cuestionarios provistos en cada formulario.

Los 13 informantes provienen de 7 caseríos (Tabla 1), y son reconocidos en su comunidad como conocedores de las plantas medicinales. Las mujeres informantes frecuentemente asisten partos en sus comunidades, y tres de los informantes masculinos conducen rituales de curación aplicando plantas medicinales y alucinógenas. Cuatro informantes reconocen su ascendencia Cocama, y otros tres descienden de padres quechua-hablantes. Ocho informantes vinieron de comunidades ubicadas en zonas inundables y cinco de comunidades ubicadas en zonas no inundables, pero su actividad diaria está ligada a las zonas inundables (Tabla 1).

En el presente trabajo registramos las plantas que son usadas como taxa ya que tratamos con varias categorías taxonómicas (especie, género y familia). Un registro de uso significa que un informante ha indicado el uso medicinal de un taxón. Una aplicación significa el uso de un taxón para un propósito particular (que puede ser modificado para uno o varios informantes). Un evento implica la interrogación de un informante sobre los usos de un taxón (en un día según Phillips, 1993).

\section{RESULTADOS}

La colaboración con los 13 informantes resultó en 750 eventos y 1164 registros de uso, aproximadamente la mitad de ellos provistos por cada sexo.

De los 170 taxas reportados como medicinales por los informantes el $40 \%$ son usados en afecciones femeninas (Tabla 2). 
La tabla 2 muestra el número de registros de uso referente a cada taxón, es decir el número de veces que han sido reportados como para usarse en afecciones de mujeres y el número de los 13 informantes que indicó el taxón como medicinal, incluyendo cualquier otro uso medicinal.

Los tres taxas con mayor registro de uso son Spondias mombin ubos (17 registros), Loranthaceae (Gén. indeterminado) suelda con suelda (14 registros) y Perseo americana palta (13 registros). El hecho de que el número de registros de uso pueda exceder el número de informantes (13) refleja que los informantes a menudo indican el mismo taxón para más de una afección femenina (por ej. aplicaciones tanto abortivas como esterilizantes). Los 67 taxas fueron reportados como útiles en afecciones femeninas en un total de 211 veces. Esto quiere decir que las afecciones femeninas constituyen el $17 \%$ de 1164 registros de uso medicinal provistos por los 13 informantes.

La Tabla 3 presenta los taxas indicados como útiles para cada uno de los 14 propósitos, organizados en las cuatro principales categorías: menstruación (A), regulación de fertilidad (13), parto (C) y enfermedades de vientre en mujeres (D). Un total de 136 aplicaciones aparecen en la Tabla 3, siendo cada uno un taxón indicado para uno de los 14 propósitos. La primera columna en la Tabla 3 (No) da el número de informantes que indican la planta para un mismo propósito. La mayoría de las aplicaciones (104 de 136) ha sido reportada solamente por uno de los 13 informantes, y el número promedio de registro de uso por aplicación solamente es $1,55(211 / 136)$. Ocho informantes reportan Persea americana palta y Gossvpium barbadense algodón para usarse como abortivos (Tabla 3-6) y como dilatador para antes del parto (Tabla 3-9), respectivamente. Este es el número más grande de registros de uso para la misma aplicación, pero muchos taxas se usan para una variedad de afecciones femeninas.

En la Tabla 2 aparece que 49 de los 67 taxas están representadas con más de un registro de uso en aflicciones de mujeres. El taxón usado con mayor amplitud es Spondias niombin ubos, habiendo sido reportado como útil para 8 de los 14 propósitos, representando todas las cuatro principales categorías sobre afecciones femeninas (Tabla 3-A,B,C,D).

Las tres columnas de la derecha en la Tabla 3 indican qué partes del taxon son preparadas para medicina, cómo se procesan y si se toma oralmente (sistemáticamente) o si se aplica externamente (tópico) al área afectada. Tratamientos sistemáticos dominan todas las 14 categorías, excepto los prolapsos de vientre (Tabla 3-13). Sin embargo, aparte de ser bebidas como decocciones e 
infusiones, también se usan frecuentemente para tomar baños. Es predominante para uso medicinal las hojas, corteza, látex y resinas (Tabla 2). Las hojas provienen de taxas cultivados, o también hierbas que se encuentran entre maleza y también en arbustos. El único árbol del bosque del cual sus hojas fueron reportadas como útiles es el Triplaris americana tangarana. La corteza, látex y resma provienen de los árboles del bosque. Una excepción importante es el uso del látex del Artocarpus altilis pan del árbol, comúnmente cultivado. Las hojas pueden ser preparadas en infusión o son hervidas para luego beber la decocción. La corteza normalmente se corta, luego se hierve y se toma la decocción o se añade aguardiente de caña de azúcar para lograr una maceración alcohólica que también se bebe. Los látex y las resinas por lo general se toman sin procesar o diluir con agua o aguardiente de caña de azúcar. Muy a menudo varios taxas se mezclan en la misma preparación medicinal y la medicina farmacológica puede ser también agregada a tales mezclas. Una decocción de semillas de Coffea arabica café con un fármaco es, por ejemplo, reportado como abortiva. El hecho de que pastillas de farmacia se mezclen en el café, aparentemente, puede explicar el efecto.

La distribución sexual de los informantes reportando usos de cada taxón también aparece en la Tabla $3(\mathrm{Ma}+\mathrm{Fe})$, y en la Tabla 4 los registros de usos provistos por los dos sexos están sumados para cada una de las 14 categorías de las afecciones femeninas, y la suma de las principales categorías (A,B,C y D) y el total de sumas y porcentajes también aparece.

Las informantes femeninas proveen 114 de los 211 registros de uso correspondiente al 5400. Esto se aproxima a la distribución total de los 1164 registros de usos medicinales con el $\mathbf{5 1 0 0}$ de ellos reportados por las seis informantes. Hay, sin embargo, mucha variación en el reporte de las principales categorías (A, B, C y D). Las informantes proveen $\mathbf{6 5 0 0}$ de los registros de uso relacionados a la menstruación (A), 68\% al control de fertilidad (B), 5100 a partos (C), pero solamente 380 o relacionados a enfermedades de vientre en mujeres (D).

La Tabla 4 muestra cuántos de los registros de uso se relacionan a los taxa de cada una de estas tres categorias: taxas predominantemente cultivados (Cult), comunes en chacras y purmas (Male) o principalmente encontrados en lugares más naturales, particularmente en el bosque (Silv), respectivamente. Los taxa cultivados (Cult) y silvestres (Silv) constituyen aproximadamente el 4000 (cada uno) de los registros de uso, y los taxa de hierbas y árboles de purmas (Male) el $\mathbf{2 0} \%$ restante. Estos porcentajes se aproximan a la distribución del hábitat de todos los 1164 registros de uso medicinal. 
Las cuatro principales categorías de uso (A, B, C y D), sin embargo, varían considerablemente (Tabla 4). De los registros de uso relacionados a la menstruación (A), control de la fertilidad (B) y partos (C), el mayor porcentaje corresponde a los taxa cultivados (Cult) y hierbas y árboles (Male) ubicados dentro y cerca del caserío, lo cual se aproxima a la distribución promedio total; mientras que para la categoría de enfermedades del vientre en mujeres, el porcentaje más alto corresponde a los árboles del bosque (Silv).

\section{DISCUSION}

Los varones pasan más tiempo que las mujeres en el bosque, razón por la cual conocen mejor los árboles. Esto explica que hay una relación causal entre los siguientes tres hechos: 1) los varones principalmente informan sobre taxas que se utilizan en enfermedades de vientre en mujeres, 2) los árboles del bosque proveen las plantas medicinales para estos males, y 3) las medicinas se preparan mayormente del látex, resma y cortezas (Tabla 3). Esto, sin embargo, no explica la preferencia por materiales de los árboles del bosque (resinas y látex) para casos de enfermedades de vientre en mujeres, en lugar de materiales de árboles y plantas cercanos a sus casas. Lo cual contrasta con el principal reporte femenino de taxas cultivados y maleza útiles en la regulación de fertilidad y para males relacionados a la menstruación y partos, lo que tiene sentido lógico. Las plantas para regulación de fertilidad pueden a menudo ser utilizadas en secreto. La presencia de taxas que tienen estos efectos en sombríos de los alrededores y en campos implica que las mujeres no tienen que depender de la extracción medicinal por medio del varón en el bosque. Además, los varones a menudo se encuentran fuera cazando, pescando o comercializando. Por eso las mujeres también necesitan fácil acceso a los taxas medicinales para estos propósitos controversiales.

En la cultura del poblador ribereño, los varones y las mujeres parecen tener un conocimiento relativamente similar sobre taxas medicinales. Los dos sexos conocen el mismo espectro de taxa medicinal pero por razones lógicas cada sexo usa y recolecta ciertas plantas con más frecuencia. A todo esto, la inclusión de informantes femeninos aparentemente no ha resultado en muchas informaciones totalmente desconocidas por los informantes masculinos, pero ha dado una información más representativa sobre los taxas medicinales de los que dependen mayormente estas comunidades. El conocimiento bastante uniforme de ambos sexos puede también reflejar que materiales de varios taxa a menudo se usan en el mismo tratamiento mezclándolos en una preparación medicinal o como curaciones suplementarias. Un varón puede proveer cortezas de un árbol del bosque y una mujer hojas de una hierba medicinal y ellos pueden, juntos, colaborar e intercambiar información. 
Los sexos también difieren en el procesamiento de plantas medicinales. Maceraciones alcohólicas son principalmente preparadas por varones, mientras que las mujeres hierven las plantas. Las maceraciones alcohólicas pueden ser preservadas por largo tiempo, en contraste a las decocciones que se deterioran y deben ser preparadas diariamente. Ya que el varón principalmente extrae material medicinal de los árboles del bosque, a menudo lejos de los caseríos, tiene sentido el hacer preparaciones que puedan ser preservadas. Por el contrario, las hierbas medicinales o plantas cultivadas pueden ser recolectadas y procesadas diariamente.

La mezcla de varios taxas en la misma preparación médica, a menudo puede dificultar la evaluación de los efectos de los taxas individuales. Al mezclar las plantas puede haber algunas que no tienen los efectos que se les atribuye. El mezclar con plantas eficientes puede también explicar rumores improbables, tales como la asignación de propiedades anticonceptivas al jugo del limón (Tabla 3-7).

Los 13 informantes demostraron conocimiento sobre plantas medicinales y evidentemente intentaron proveer informaciones confiables. A pesar de eso, algunos registros de uso probablemente reflejen rumores, identificaciones equivocadas y plantas de selección secundaria en prácticas que se usaron raramente o tal vez nunca. Por ej. Achyranthus aspera y Priva lappulaceo amor seco, fueron indicadas como alternativas menos eficientes que el Desmodiurn adscendens amor seco (Tabla 3-7, 8). Es, por lo tanto, importante estudiar las prácticas medicinales de varias personas. Los taxa indicados como útiles repetidamente por informantes independientes parecen, por lo general, más probables de tener efectos fisiológicos. También es importante darse cuenta que no existe un limite máximo en el número de plantas a las que se les atribuye propiedades medicinales. Un estudio continuado con más informantes proveería taxas adicionales mayormente poco conocidas para males femeninos y otros propósitos medicinales. Registro de usos dados por solamente un informante no debería ser de mucha importancia, pero tampoco debería ser desechado inmediatamente por ineficiente. Un estudio de la literatura puede proveer similares usos de alguna otra parte. Un caso instructivo es el uso de semillas de Persea americana palta, para control de fertilidad. Uno de los autores del presente estudio ha investigado previamente la etnobotánica de los indígenas en el noroeste del Ecuador (Kvist \& HolmNielsen, 1987; Barfod \& Kvist, 1996), encontrando una familia que indicó que las mujeres utilizan las semillas de palta como esterilizante. Esta información parece improbable hasta que se llegue a aclarar que la semilla de palta es usada en la misma forma por los nativos americanos Siona-Secoya en la Amazonía del Ecuador (Kvist \& Holm-Nielsen, 1987), y los indios Tikunas en la Amazonía colombiana (Glenboski, 1983). Las informaciones repetidas de efectos abortivos en el presente estudio (Tabla 3-6) hace altamente probable que la semilla de Persea americana palta, contenga propiedades de control de fertilidad. Otra similitud entre los datos etnomedicinales del noroeste del Ecuador es el uso del género Desmodium 
como un abortivo, pero en Ecuador es usado el Desmodiumi uncinatum (Kvist \& HolmNielsen, 1987) y en el área del presente estudio es usado Desmodim adscendens.

Especies relacionadas a menudo contienen sustancias similares causando los mismos efectos. Por eso la literatura también debería ser estudiada para usos similares de especies relacionadas.

Un estudio etnomedicinal ideal debería resultar en determinaciones botánicas confiables de todas las plantas indicadas para ser usadas, y una estimación sustancial de la importancia relativa de las plantas y habitats aplicada a la comunidad. Hay un conflicto inherente entre estos dos objetivos, ya que algunas plantas son mucho más difíciles de encontrar y recolectar que otras, y una demanda consistente por verificadores botánicos puede distorsionar los resultados. Especies sembradas y de entre maleza llegan a ser sobrerrepresentadas y la importancia de los árboles del bosque y lianas desestimadas. En el presente estudio identificamos hasta donde fue posible, a especies científicas, pero aceptamos una incertidumbre referente a la identidad de algunas plantas. Renaquilla, por ejemplo, es una planta medicinal importante y todas nuestras colecciones verificadas han probado que provienen dc pequeños árboles de higuera Ficus spp (Tabla 2). ya que algunos ribereños también llaman «renaquilla» a la Coussapoa y Clussia; estos géneros pueden ocasionalmente ser usados medicinalmente para los mismos propósitos que las especies Ficus. Para poder apreciar la importancia de la «renaquilla» tenemos que aceptar que algunos de los registros de uso indocumentados pueden presentar otros géneros. Dc acuerdo con la realidad de los informantes, esto botánicamente distingue a los matapalos; sin embargo, todo representa a las mismas especies tradicionales.

\section{CONCLUSIONES}

1. Varones y mujeres reportan relativamente en forma equitativa acerca de plantas útiles para males en mujeres, pero las mujeres proveen más registros de uso relacionados a la regulación de fertilidad y problemas de menstruación, y los varones más registros de uso relacionados a enfermedades de vientre en mujeres.

2. Las mujeres principalmente extraen material medicinal de plantas de cultivo y de entre maleza, y los varones de los árboles del bosque. De plantas cultivadas se usan mayormente hojas que se procesan por hervor; y de árboles del bosque, cortezas que se procesan bajo maceraciones alcohólicas así como látex y resinas.

3. Varones y mujeres comparten la mayor parte de sus conocimientos sobre plantas medicinales; las diferencias en el reporte de frecuencias se deben generalmente a que cada sexo, por razones lógicas, principalmente colectan y usan ciertos taxas para curar males particulares. 
4. Numerosas plantas se reportan como útiles pero pocas se reportan repetidamente para el mismo propósito, las últimas son las más probables de tener efectos fisiológicos, pero las anteriores pueden también ser eficientes, particularmente silos mismos usos se reportan de otro lugar.

5. Para conseguir una impresión concisa de qué plantas y habitats principalmente se usan para medicina, es necesario aceptar una incertidumbre concerniente a la identidad científica exacta de algunas especies tradicionales.

\section{AGRADECIMIENTOS}

Agradecemos a la población de los caseríos en la zona de Jenaro Herrera, por su generosa hospitalidad y particularmente a nuestros 13 informantes, quienes nos comunicaron sus conocimientos y experiencias. A Fernando Huayta, por ser un invalorable recurso de contacto, y a Arístides Vásquez por su trabajo entusiasta, proveyendo el soporte logístico a pesar de las dificultades de las condiciones de trabajo. Al equipo del Centro de Investigaciones de Jenaro Herrera $(\mathrm{CJJH})$, por proporcionar un placentero y estimulante ambiente para nuestro trabajo.

En Iquitos, al equipo y administración del Instituto de Investigaciones de la Amazonía Peruana (IIAP) y al Herbario (AMAZ) de la Universidad Nacional de la Amazonía Peruana (UNAP), por su ayuda para resolver muchos problemas técnicos.

A Gustav Nebel, por la elaboración del mapa del área de estudio.

El Blgo. Kember Mejía revisó el manuscrito y aportó sugerencias para la presentación del trabajo.

Los estudios fueron financiados por la Danish International Developing Agency (Danida). 


\section{BIBLIOGRAFIA}

BARFOD, A. \& KVIST, L. P. 1996. Comparative ethnobotanical studies of the Amerindian groups in Coastal Ecuador. The Royal Danish Academy of Sciences andLetters. En: Biological Papers 46: 1-166.

CHIBNIK, M. \& JONG, W. DE. 1992. Organización de la mano de obra agrícola en las comunidades ribereñas de la Amazonia Peruana. En: Amazonía Peruana.21: 181-215.

GLENBOSKI, L. G. 1983. Ethnobotany of the Tikuna Indians, Amazonas, Colombia. BibliotecaJ. J. Triana4: 1-92. Universidad Nacional de Colombia, Bogotá.

HERN, W. M. 1994. Conocimiento y uso de anticonceptivos herbales en una comunidad shipibo. En: Amazonía Peruana 24: 143-160.

HIRAOKA, M. 1985. Mestizo subsistence in riparian Amazonía. En: NationaL Geographic Research 1: 23 6-246.

KAINER, K. A. \& DURYEA, M. L. 1992. Tapping womens knowledge: Plantresource ji use in extractive reserves, Acre, Brazil. En: Economic Botany 46: 408-425.

K.AHN, F. 1988. Ecology of economically important palms in Peruvian Amazonía. En: Advances in Economic Botany 6: 42-49.

\& MEJIA, K. 1990. Palm communities in wetland forest ecosystems of Puvian Amazonía. En: Forest Ecology and Management 33/34: 170-179.

KVIST, L. P. A comparison of qualitative and three quantitative ethnomedicinal methodologies based on studies in Peru and Ecuador. Memorias del segundo Simposio Ecuatoriano de Etnobotónica y Botónica Económica. Quito (Ecuador): (lo Press).

\& HOLM-NIELSEN, L. B. 1987. Ethnobotanical aspects of lowland Ecuador. En: Opera Botan ica 92: 83-107.

MEJIA, C. 1988. Utilization of palms in eleven mestizo villages of the Peruvian Amazon (Ucayali river, department of Loreto). En: Advances in Economic Botany 6: 130136. 
PADOCH, C. \& JONG, DE W. 1989. Production and profit in agroforestry: An example from the Peruvian Amazon. En: Browder, JO. (ed.), Fragile lands of Latin American. Boulder, San Fransisco, \& London: Westview Press. pp.

102-113.

1 990. Santa Rosa: The impact of the forest products trade on Amazonian place and population. En: Advances in Economic Botany 8: 151-158.

1991. The house gardens of Santa Rosa: Diversity and variability in an Amazonian Agricultural System. En: Economic Botany 45: 166-175.

PARODI, J. L. 1988. The use of palms and other native plants in non-conventional low cost rural housing in the Peruvian Amazon. En: Advances in Economic Botany6: 119-129.

\& FREITAS, D. 1990. Geographical aspects of forested wetlands in the lower Ucayali, Peruvian Amazonía. En: Forest Eco logy and Management 33/34: 157-168.

PETERS, C. M. \& HAMMOND, E. J. 1990. Fruits from the flooded forests of Peruvian Amazonía: Yield estimates for natural populations of three promising species. En: Advances in Economic Botany 8: 159-176.

PHILLIPS, O. \& GENTRY A. H. 1993. The useful plants of Tambopata, Pem: 1. Statistical hypotheses tests with a new quantitative teehnique. En: Economic Botany 47: 15-32.

RIVAS, R. 1994. La mujer Cocama del bajo Ucayali: Matrimonio, embarazo, parto y salud. En: Amazonía Peruana 24: 227-242.

TOURNON, J. 1984. Investigaciones sobre las plantas medicinales de los Shipibo Conibo del Ucayali. En: Amazonía Peruana 10: 91-118. 
Tabla 1. Informantes en el estudio etnomedicinal numerados de acuerdo con el orden en que participaron. La relación de informantes presenta sexo y edad del informante, caserío de residencia, hábitat del caserío (A: Zona no inundable. B: Zona inundable) y lugar de nacimiento del informante.

\begin{tabular}{lllll}
\hline Sexo & Edad & Caserío & Hábitad & Nacido \\
\hline 1. Masculino & 69 & Yanallpa & $\mathrm{B}$ & Río Napo \\
2. Masculino & 62 & Yanallpa & $\mathrm{B}$ & Río Huallaga \\
3. Femenino & 56 & Jenaro Herrera & $\mathrm{A}$ & Jorge Chávez \\
4. Masculino & 33 & Jenaro Herrera & $\mathrm{A}$ & Jenaro Herrera \\
5. Masculino & 35 & Iricahua & $\mathrm{B}$ & Iricahua \\
6. Femenino & 65 & Nuevo Aucayacu & $\mathrm{A}$ & Pumacahua \\
7. Femenino & 59 & Yanallpa & $\mathrm{B}$ & Yanallpa \\
8. Masculino & 55 & Casa Grande & $\mathrm{B}$ & Iricahua \\
9. Femenino & 48 & Casa Grande & $\mathrm{B}$ & Casa Grande \\
10. Femenino & 37 & Nuevo Aucayacu & $\mathrm{A}$ & No registrado \\
11. Masculino & 67 & Jenaro Herrera & $\mathrm{A}$ & Canal de Puinahua \\
12. Masculino & 38 & Nuevo San Juan & $\mathrm{B}$ & Jenaro Herrera \\
13. Femenino & 52 & Progreso & $\mathrm{B}$ & Iquitos \\
\hline
\end{tabular}




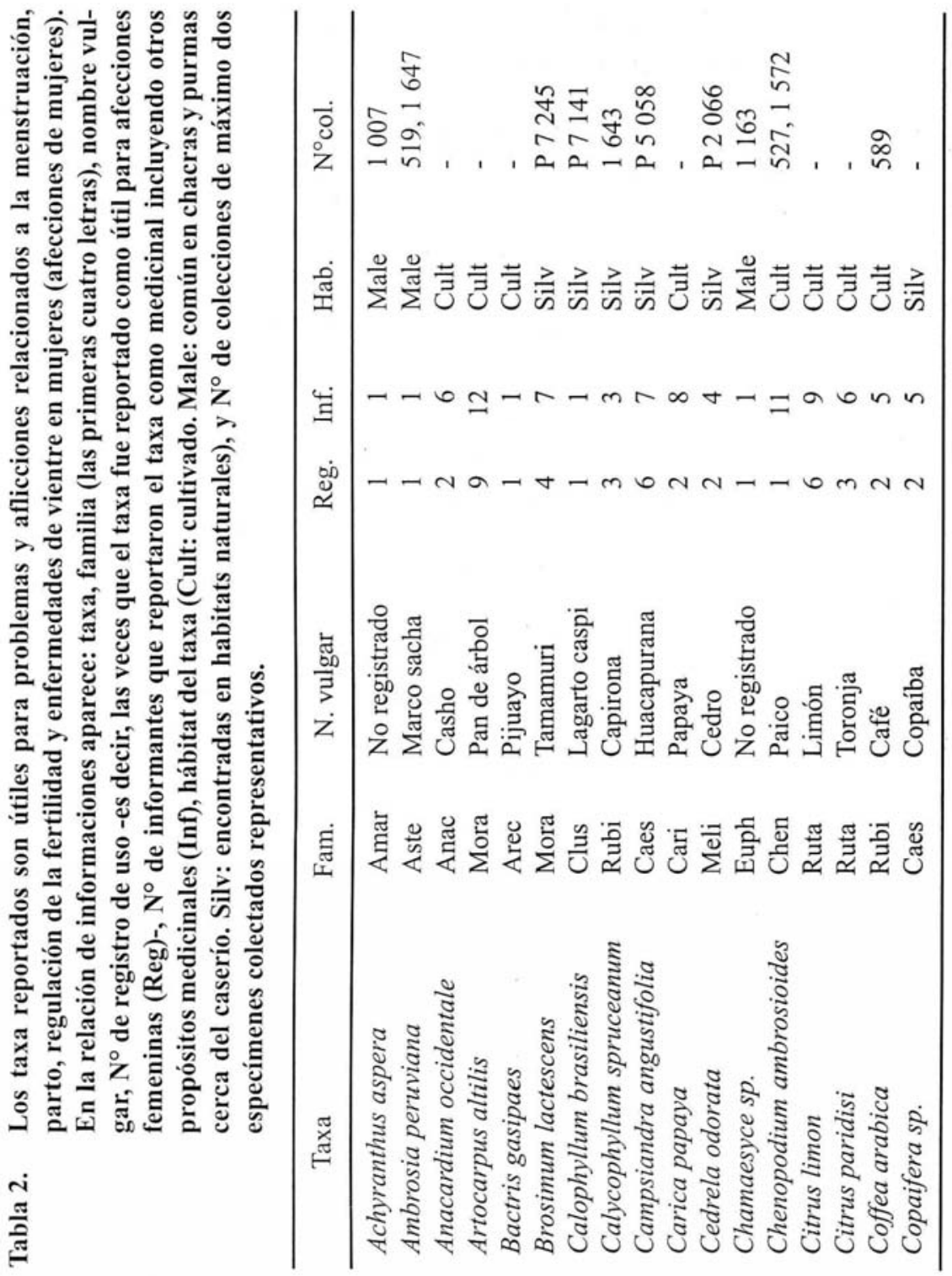




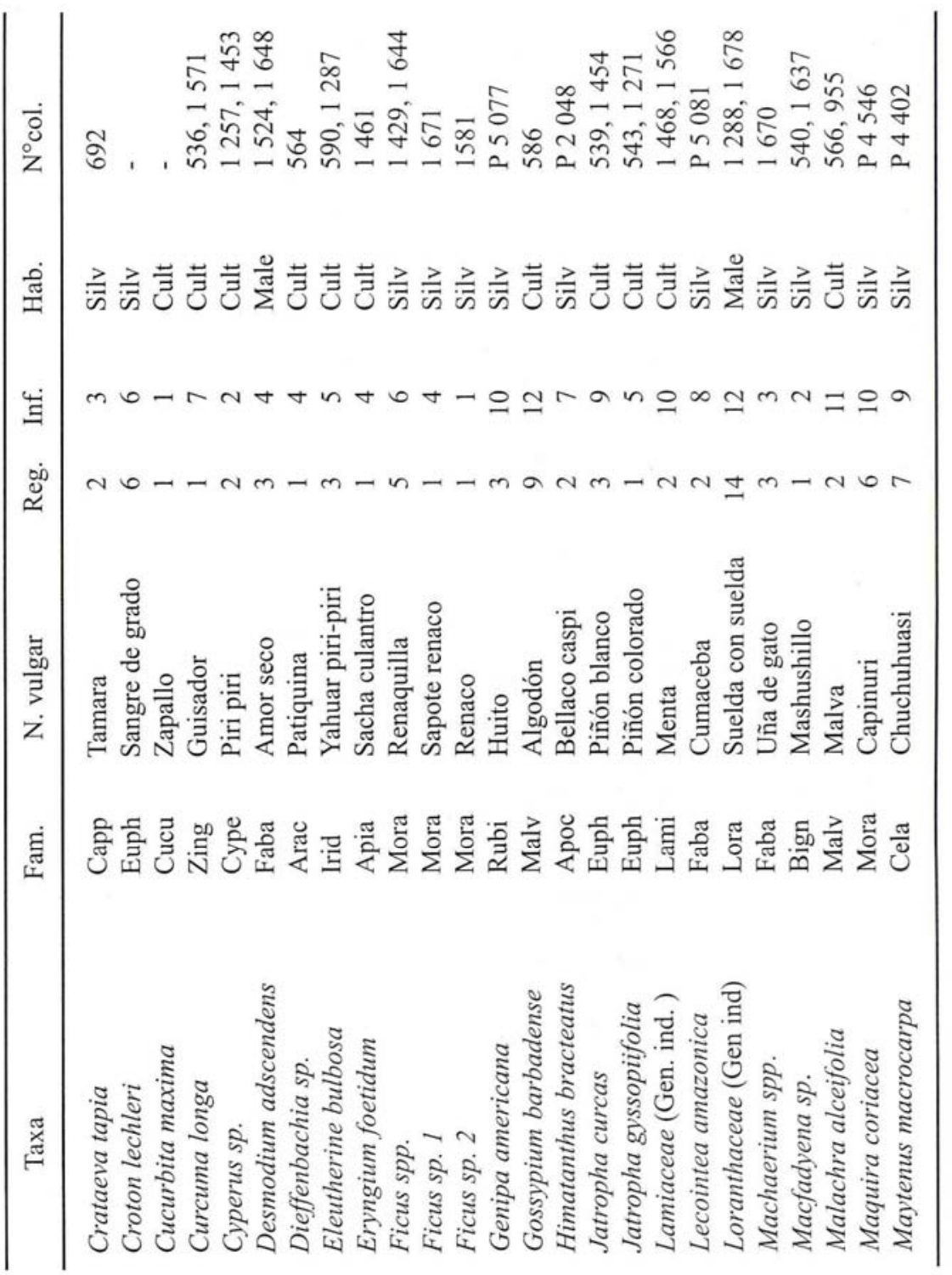




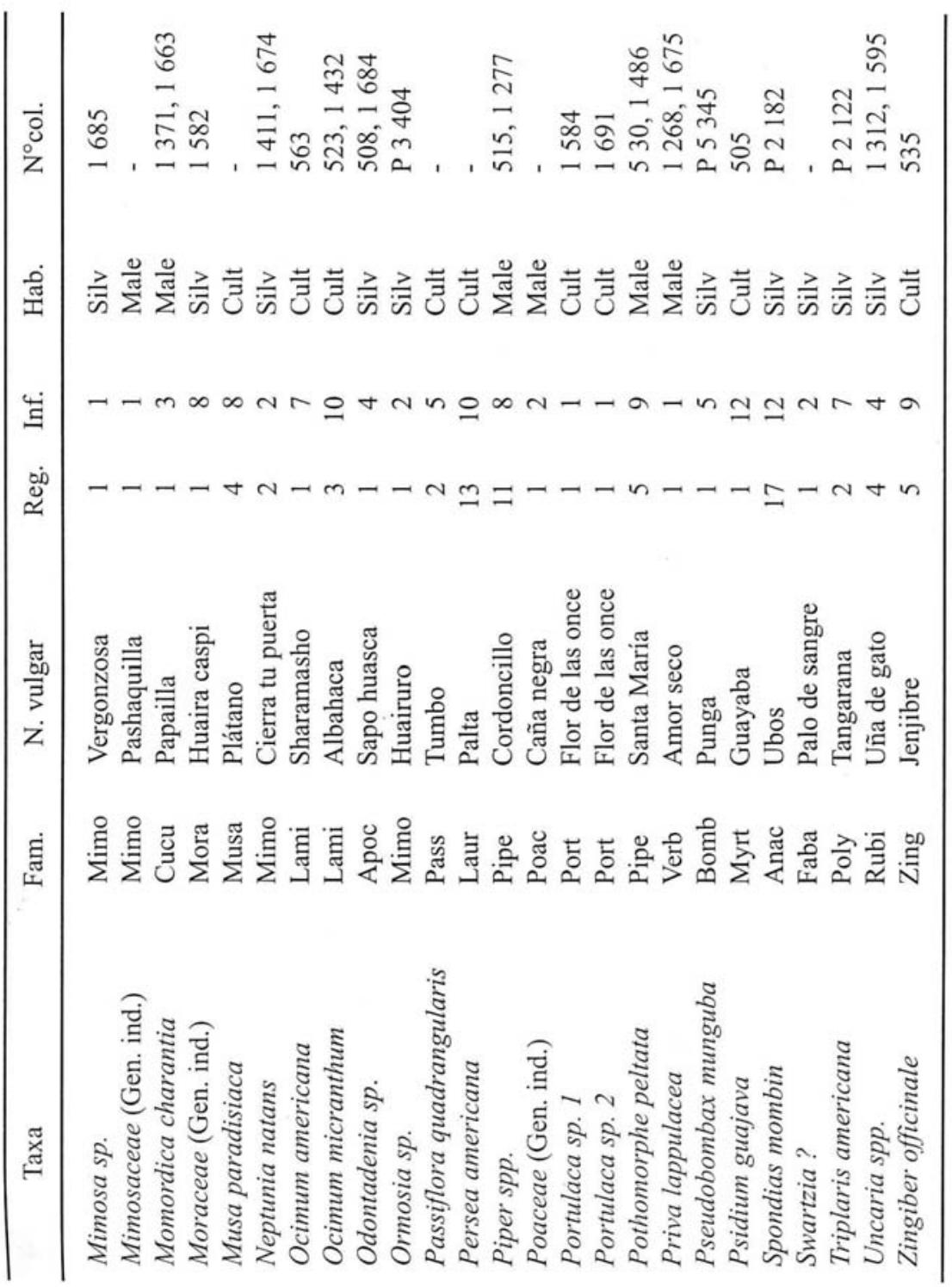


Tabla 3. Los taxa usados para cada uno (le los 14 propósitos relacionados con problemas y aflicciones de mujeres están organizados en categorías principales: Menstruación (A), Control de la fertilidad (B), Parto (C) y Enfermedades (tel vientre en mujeres (D).

\begin{tabular}{|c|c|c|c|c|c|}
\hline Cat. De uso/taxa & No & $\mathrm{Ma}+\mathrm{Fe}$ & Part. & Proceso & Trat. \\
\hline \multicolumn{6}{|l|}{ A. Mentruación } \\
\hline $\begin{array}{l}\text { 3-1.Menstruación(regulació } \\
\text { Persea americana } \\
\text { Artocarpus altilis } \\
\text { Campsiandra angustijolia } \\
\text { Ctirus paradisi } \\
\text { Croton Lechleri } \\
\text { Cyperus sp. } \\
\text { Himatanthus bracteatus } \\
\text { Jatropha curcas } \\
\text { Jatropha gyssopitfolia } \\
\text { Malachra alciefolia } \\
\text { Piper spp. } \\
\text { Spondias momibin } \\
\text { Zingiber officinale }\end{array}$ & $\begin{array}{l}\text { 3: } \\
\text { 1: } \\
\text { 1: } \\
\text { 1: } \\
\text { 1: } \\
\text { 1: } \\
\text { 1: } \\
1: \\
1: \\
1: \\
1: \\
1: \\
1:\end{array}$ & $\begin{array}{l}0+3 \\
0+1 \\
1+0 \\
1+0 \\
0+1 \\
0+1 \\
0+1 \\
0+1 \\
0+1 \\
1+0 \\
0+1 \\
0+1 \\
0+1\end{array}$ & $\begin{array}{l}\text { Sern } \\
\text { Lát } \\
\text { Cor } \\
\text { Fru } \\
\text { Res } \\
\text { BuI } \\
\text { Hoj } \\
\text { Hoj } \\
\text { Hoj } \\
\text { Flo } \\
\text { Hoj } \\
\text { Cor } \\
\text { Bul }\end{array}$ & $\begin{array}{l}\text { Decocción } \\
\text { No proc. } \\
\text { Decocción } \\
\text { No proc. } \\
\text { No proc. } \\
\text { Decocción } \\
\text { Decocción } \\
\text { Macerac. } \\
\text { Macerac. } \\
\text { Decocción } \\
\text { Decocción } \\
\text { Decocción } \\
\text { Decocción }\end{array}$ & $\begin{array}{l}\text { sy } \\
\text { sy } \\
\text { sy } \\
\text { sy } \\
\text { sy } \\
\text { sy } \\
\text { to } \\
\text { to } \\
\text { to } \\
\text { sy } \\
\text { sy } \\
\text { sy/to } \\
\text { sy }\end{array}$ \\
\hline \multicolumn{6}{|c|}{ 3-2. Dolores abdominales (durante la menstruación): } \\
\hline $\begin{array}{l}\text { Spondias mombin } \\
\text { Artocarptis altilisis } \\
\text { Genipa americana } \\
\text { Jatropha curcas } \\
\text { Lamiaceae (Gen. md.) } \\
\text { Loranthaccae (Gen. md.) } \\
\text { Mac fadyena sp. } \\
\text { Maquira coriacea } \\
\text { Piper spp. }\end{array}$ & $\begin{array}{l}2: \\
1: \\
1: \\
1: \\
1: \\
1: \\
1: \\
1: \\
1:\end{array}$ & $\begin{array}{l}2+0 \\
0+1 \\
0+1 \\
0+1 \\
0+1 \\
1+0 \\
0+1 \\
0+1 \\
0+1\end{array}$ & $\begin{array}{l}\text { Cor } \\
\text { Lat } \\
\text { Cor } \\
\text { Res } \\
\text { Hoj } \\
\text { Hoj } \\
\text { Hoj } \\
\text { Lat } \\
\text { Hoj }\end{array}$ & $\begin{array}{l}\text { Decocción } \\
\text { No proc. } \\
\text { No registrade } \\
\text { No proc. } \\
\text { Decocción } \\
\text { Decocción } \\
\text { Decocción } \\
\text { No proc. } \\
\text { Decocción }\end{array}$ & $\begin{array}{l}\text { sy } \\
\text { sy } \\
\text { to } \\
\text { sy } \\
\text { sy } \\
\text { sy } \\
\text { sy } \\
\text { sy } \\
\text { sy }\end{array}$ \\
\hline
\end{tabular}




\begin{tabular}{|c|c|c|c|c|c|}
\hline Cat. de uso/taxa & No & $\mathrm{Ma}+\mathrm{Fe}$ & Parts. & Proceso & Trat. \\
\hline \multicolumn{6}{|c|}{ 3-3. Hemorragias (violentas/ prolongadas): } \\
\hline Spondias mombin & 2: & $2+0$ & Cor & Decocción & sy/to \\
\hline Eleutherine bulbosa & 1: & $1+0$ & Bul & Decocción & sy \\
\hline Musa paradisíaca & 1: & $1+0$ & Cás & Decocción & sy \\
\hline Piper spp. & 1: & $1+0$ & Hoj & Decocción & sy \\
\hline Swartzia? & 1: & $1+0$ & Cor & Decocción & sy \\
\hline \multicolumn{6}{|c|}{ B. Regulación de la fertilidad: } \\
\hline \multicolumn{6}{|c|}{ 3.4. Embarazo (para promover) } \\
\hline Canipsiandra angusufolia & 1: & $1+0$ & Raíz & Mezcla & sy \\
\hline Persea americana & 1: & $0+1$ & Hoj & Macerac. & to \\
\hline Poaceae (Gen. md.) & 1: & $0+1$ & Ta11 & Decocción & sy \\
\hline \multicolumn{6}{|l|}{ 3.5. Aborto (para prevenir) } \\
\hline Bactris gasipaes & 1: & $0+1$ & Raíz & Decocción & sy \\
\hline Campsiandra angustifolia & 1: & $0+1$ & Cor & Infusión & sy \\
\hline Lecointea amazónica & 1: & $1+0$ & Cor & Decocción & sy \\
\hline \multicolumn{6}{|l|}{ 3-6. $\quad$ Abortivos: } \\
\hline Persea americana & 8: & $3+5$ & Sem & Decocción & sy \\
\hline Citrus limon & 5: & $3+2$ & Raíz & Decocción & sy \\
\hline Genipa americana & 2: & $2+0$ & Fru & Infusión & sy \\
\hline Passiflora quadrangularis & 2: & $1+1$ & Hoj & Decocción & sy \\
\hline A nacardium occiden tale & 1: & $1+0$ & Sem & Decocción & sy \\
\hline Carica papaya & 1. & $0+1$ & Flor & Decocción & sy \\
\hline Citrus paradisi & 1: & $0+1$ & Raiz & Decocción & sy \\
\hline Coffea arabica & 1: & $1+0$ & Sem & Decocción & sy \\
\hline Curcuma longa & 1: & $1+0$ & Bul & Decocción & sy \\
\hline Dieffenbachia sp. & 1: & $0+1$ & Hoj & Macerac. & to/sy \\
\hline Jatropha curcas & 1: & $1+0$ & Sem & Infusión & sy \\
\hline
\end{tabular}




\begin{tabular}{|c|c|c|c|c|c|}
\hline Cat. de uso/taxa & No & $\mathrm{Ma}+\mathrm{Fe}$ & Parts. & Proceso & Trat. \\
\hline Momordica charantic & 1: & $0+1$ & Hoj & Decocción & Sy \\
\hline Aforaceae (Gen. md.) & 1: & $1+0$ & Lát & No Proc. & Sy \\
\hline Ormosia sp. & 1: & $1+0$ & Sem & Decocción & Sy \\
\hline Portulaca sp. & 1: & $0+1$ & Hoj & Dcc occ ión & Sy \\
\hline Pothomorphe peltata & 1: & $0+1$ & Hoj & Decocción & Sy \\
\hline Triplaris americana & 1: & $0+1$ & Cor & Decocción & sy \\
\hline \multicolumn{6}{|l|}{ 3-7 Anticonceptivos } \\
\hline Loranthaceae (Gen. md.) & 3: & $0+3$ & Hoj & Decocción & sy \\
\hline Mimosa sp. & 1: & $1+0$ & Raíz & Decocción & sy \\
\hline Priva lappulacea & 1: & $0+1$ & Hoj & No registrado & sy \\
\hline Neptuna natans & 1: & $0+1$ & Hoj & Decocción & sy \\
\hline Calycophyllum spruceanum & 1: & $0+1$ & Cor & Decocción & sy \\
\hline Desmodium adscendens & 1: & $0+1$ & Raíz & Decocción & sy \\
\hline Carica papaya & 1: & $0+1$ & Flor & Decocción & sy \\
\hline Pothomorphe peltata & 1: & $0+1$ & Hoj & Decocción & sy \\
\hline Citrus liman & 1: & $0+1$ & Fru & No registrado & sy \\
\hline Zingiber officinale & 1: & $0+1$ & Bul & Decocción & sy \\
\hline Chenopodium ambrosioides & 1: & $0+1$ & Hoj & Decocción & sy \\
\hline Ocimum micranthum & 1: & $0+1$ & Hoj & Decocción & sy \\
\hline Psidium guajava & 1: & $0+1$ & Sem & Infusión & sy \\
\hline Spondias mombin & 1: & $1+0$ & Cor & Decocción & sy/ to \\
\hline \multicolumn{6}{|l|}{ 3-8 Esterelizante: } \\
\hline Loranthacae (Gen. md.) & 3: & $0+3$ & Hoj & Decocción & sy \\
\hline Achyranthuas aspera & 1: & $0+1$ & Hoj & Decocción & sy \\
\hline Crataeva tapia & 1: & $1+0$ & Cor & No registrado & to \\
\hline Croton lechleri & 1: & $0+1$ & Res & No proc. & sy \\
\hline Desmodium adscendens & 1: & $0+1$ & Hoj & Decocción & sy \\
\hline Mimosaceae (Gen. md.) & 1: & $0+1$ & Hoj & Infusión & sy \\
\hline Neptunia natans & 1: & $0+1$ & Hoj & Decocción & sy \\
\hline Ocimum americana & 1: & $0+1$ & Hoj & Decocción & sy \\
\hline Piper spp. & 1: & $0+1$ & Hoj & Decocción & sy \\
\hline
\end{tabular}




\begin{tabular}{|l|l|l|l|l|l|}
\hline Cat. de uso/taxa & No & Ma+Fe & Parts. & Proceso & Trat. \\
\hline Portulacada sp.2 & $1:$ & $1+0$ & Hoj & Decocción & sy \\
Triplaris americana & $1:$ & $0+1$ & Hoj & Decocción & sy \\
Zingiber officinale & $1:$ & $0+1$ & Bull & Decocción & sy \\
& & & & & \\
\hline
\end{tabular}

\section{Parto}

\section{3-9 Dilatador (antes del parto):}

Gossypium barbadense

Musa paradisíaca

Piper spp.

Ocimum micranthum

Citrus paradisi

Eryngium foetida

Lamiaceae (Gen. md.)

Persea americana

\begin{tabular}{|l|l|l|l|l}
\hline$:$ & $4+4$ & Hoj & Decocción & sy \\
3 & $2+1$ & Hoj & Decocción & sy \\
3 & $2+1$ & Hoj & Decocción & sy/to \\
2 & $0+2$ & Hoj & Decocción & sy \\
$1:$ & $1+2$ & Hoj & Decocción & sy \\
$1:$ & $0+1$ & Hoj & Decocción & sy \\
$1:$ & $0+1$ & Hoj & Decocción & sy \\
$1:$ & $1+0$ & Hoj & Decocción & sy \\
& & & &
\end{tabular}

\section{3-10. Placenta (eliminación):}

\begin{tabular}{|l|l|l|l|l|l|}
\hline $\begin{array}{l}\text { Pothomorphe peltata } \\
\text { Chamaesyce sp. }\end{array}$ & $\begin{array}{l}2: \\
1:\end{array}$ & $0+2$ & Hoj & Decocción & sy \\
Decocción & sy
\end{tabular}

\section{3-1. Sobreparto:}

\begin{tabular}{|l|l|l|l|l|l|} 
Campsiandra angustifolia & $2:$ & $2+0$ & Cor & Infusión & sy \\
Eleutherine bulbosa & $2:$ & $1+1$ & Bul & Decocción & sy \\
Piper spp. & $2:$ & $1+1$ & Hoj & Decocción & sy \\
Zingiber officinale & $2:$ & $2+0$ & Bul & Decocción & sy \\
Ambrosía peruviana & $1:$ & $0+1$ & Hoj & Decocción & sy/to \\
Coffea arabica & $1:$ & $0+1$ & Hoj & Decocción & sy \\
Cyperus sp. & $1:$ & $0+1$ & Bul & Infusión & sy \\
Gossypium barbadense & $1:$ & $1+0$ & Hoj & Decocción & to \\
Lecoin tea amazoníca & $1:$ & $0+1$ & Cor & Infusión & sy \\
Loranthacae (Gen. md.) & $1:$ & $0+1$ & Hoj & Macerac. & sy/to \\
Maytenus macrocarpa & $1:$ & $1+0$ & Cor & Infusión & sy \\
& & & & &
\end{tabular}




\begin{tabular}{|c|c|c|c|c|c|}
\hline Cat. de uso/taxa & No & $\mathrm{Ma}+\mathrm{Fe}$ & Parts. & Proceso & Trat. \\
\hline Pothomorphe peltata & 1: & $0+1$ & Raíz & Decocción & sy \\
\hline Psudobombax munguba & 1: & $1+0$ & Flor & Infusión & sy \\
\hline Spondias mombin & 1: & $1+0$ & Cor & Decocción & sy /to \\
\hline \multicolumn{6}{|c|}{ D. Enfermedades de Vientre en mujeres: } \\
\hline \multicolumn{6}{|c|}{ 3-12. Heridas en la Vagina: } \\
\hline Spondias mombin & 6: & $3+3$ & Cor & Decocción & to \\
\hline Artocarpus altilis & 5: & $4+1$ & Lát & No proc. & sy \\
\hline Ficus spp. & 5: & $2+3$ & Lát & No proc. & sy/to \\
\hline Maytenus macrocarpa & 5: & $3+2$ & Cor & Decocción & sy \\
\hline Loranthacae (Gen. md.) & 4: & $2+2$ & Hoj & Decocción & sy \\
\hline Maquira coriacea & 4: & $3+1$ & Lát & No proc. & sy \\
\hline Uncaria spp. & 4: & $2+2$ & Cor & Decocción & sy \\
\hline Brosimum lactescens & 3: & $2+1$ & Lát & No proc. & sy/ to \\
\hline Croton lechleri & 3: & $2+1$ & Res & No proc. & sy \\
\hline Machaerium spp. & 3: & $2+1$ & Cor & Decocción & sy/ to \\
\hline Calycophyllum spruceanum & 2: & $0+2$ & Cor & Decocción & sy/ to \\
\hline Copaifera sp. & 2: & $0+2$ & Res & No proc. & sy \\
\hline Anacardíum occidentale & 1: & $1+0$ & Cor & Decocción & to \\
\hline Campsiandra angustifolia & 1: & $0+1$ & Cor & Infusión & sy \\
\hline Cedrela odorata & 1: & $1+0$ & Cor & Decocción & sy \\
\hline Crataeva tapia & 1: & $1+0$ & Cor & Decocción & sy \\
\hline Cucurbita maxima & 1: & $0+1$ & Fru & Infusión & sy \\
\hline Desmodium adscendens & 1: & $0+1$ & Hoj & Decocción & sy \\
\hline Ficus sp. 1 & 1: & $1+0$ & Lát & No proc. & sy \\
\hline Ficus sp. 2 & 1: & $1+0$ & Cor & Infusión & sy \\
\hline Himatanthus bracteatus & 1: & $1+0$ & Lát & Infusión & sy/ to \\
\hline Odontadenia sp. & 1: & $1+0$ & Lát & Infusión & sy \\
\hline Piper spp. & 1: & $1+0$ & Hoj & Decocción & sy \\
\hline \multicolumn{6}{|l|}{ 3-13. Prolapso abdominal: } \\
\hline Calophyllum brasiliensis & 1: & $0+1$ & Hoj & No proc. & to \\
\hline
\end{tabular}




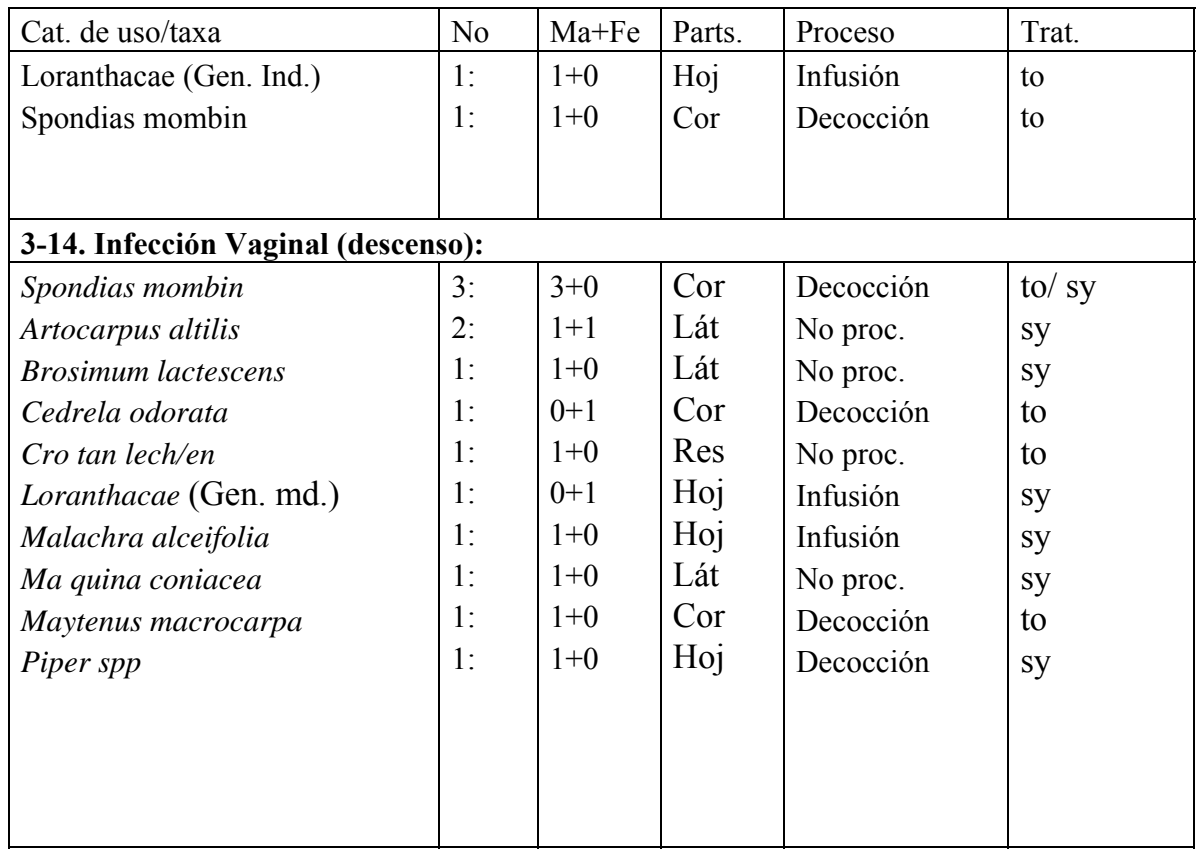

La relación de informaciones presenta: $\mathrm{N}^{\circ}$ de informantes mencionando el uso (No) la distribución sexual de estos informantes $(\mathrm{Ma}+\mathrm{Fe})$, qué parte de la planta es preparada para medicina (Part.), cómo es preparada (Proceso), y si el uso es sistemático (sy) o tópico (to), es decir, si es bebido o aplicado por fuera, respectivamente. 
Tabla 4. Registros de uso proporcionados por los dos sexos, y habitats de los taxas medicinales de acuerdo con el número de registros de liso. No: número total (le registros de uso proporcionados para un propósito particular. Ma+Fe: números de estos registros de uso, proporcionados por informantes masculinos $y$ femeninos, respectivamente. \%Fe: porcentaje de registros de uso proporcionados por informantes femeninos (perteneciente a las 4 principales categorías de uso $\mathrm{A}, \mathrm{B}, \mathrm{C}$ y $\mathrm{D}$ ). Cult: número de registro de uso relacionados a taxa que son cultivados. tVIale: registros de uso relacionado a taxas principalmente encontrados en chacras y purmas jóvenes. Silv: taxa encontrados en habitats más naturales, particularmente bosques. \%Cas: porcentaje de registros de uso relacionados a taxas principalmente encontrados cerca de los caseríos (Cult, Male), en contraste con los que se encuentran en el bosque (Silv).

\begin{tabular}{|l|l|l|l|l|l|l|l|l|}
\hline Propósito de uso & No & $\mathbf{M a}+$ & Fe & \%Fe & Cult & Male & Silv. & \% Cas \\
\hline A. 1. Menstruación. & $15:$ & 3 & 12 & & & & & \\
2. Dolores abdominales & $10:$ & 3 & 7 & & & & & \\
$\begin{array}{l}\text { 3. Hemorragia Suma } \\
\text { (menstruación) }\end{array}$ & $6:$ & 5 & 1 & & & & & \\
& $3: 1$ & 11 & 20 & 65 & 16 & 4 & 11 & 55 \\
\hline B. 4. Fertilizantes & $3:$ & 1 & 2 & & & & & \\
5. Antiabortivos & $3:$ & 1 & 2 & & & & & \\
6. Abortivos & $30:$ & 15 & 15 & & & & & \\
7. Anticonceptivos & $16:$ & 2 & 14 & & & & & \\
8. Esterilizantes & $14:$ & 2 & 12 & & & & & \\
$\quad$ Suma (fertilidad) & $66:$ & 21 & 45 & 68 & 35 & 16 & 15 & 76 \\
& & & & & & & & \\
\hline C. 9. Dilatador (antes) & $20:$ & 10 & 10 & & & & & \\
10.Eliminar placenta & $3:$ & 0 & 3 & & & & & \\
11. Después del parto & $18:$ & 10 & 8 & & & & & \\
$\quad$ Suma (parto) & $41:$ & 20 & 21 & 51 & 24 & 11 & 6 & 80 \\
\end{tabular}




\begin{tabular}{|l|l|l|l|l|l|l|l|l|}
\hline Propósito de uso & No & Ma+ & Fe & \%Fe & Cult & Male & Silv. & \% Cas \\
\hline D. 1. Heridas en vagina & 57 & 33 & 24 & & & & & \\
2. Prolapso Abdominal & 3 & 2 & 1 & & & & & \\
3. Infección Vaginal & 13 & 10 & 3 & & & & & \\
$\quad$ Suma (aflicc. Abd.) & 73 & 45 & 28 & 38 & 10 & 9 & 54 & 26 \\
\hline $\begin{array}{l}\text { Suma (total) } \\
\text { Porcentaje (del total) }\end{array}$ & 21 & 46 & 54 & 54 & 40 & 19 & 41 & 59 \\
& & & & & & & & \\
\hline
\end{tabular}


Anexo 1: Mapa de ubicación del área de estudio.

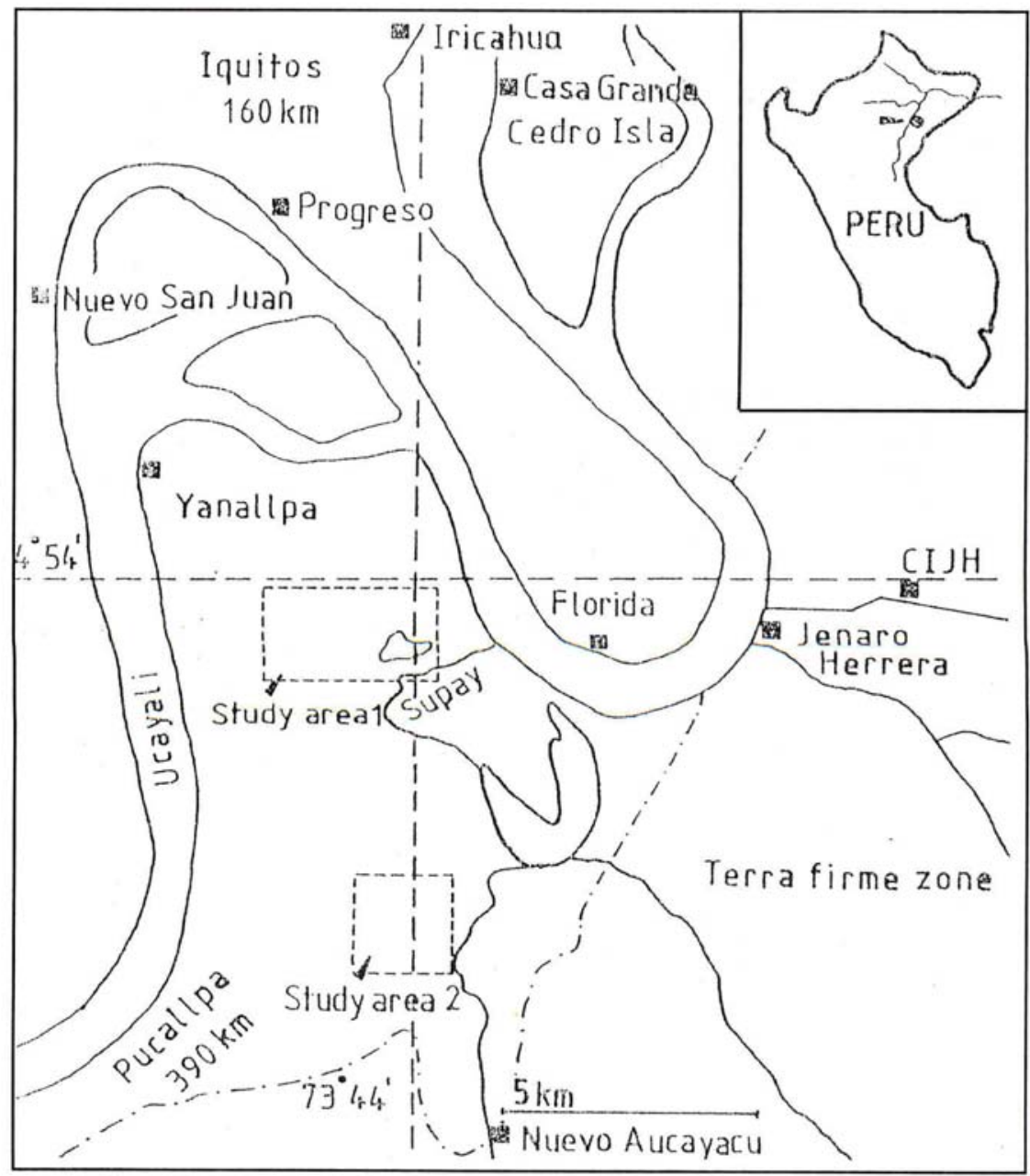


Anexo 2. Cuestionario aplicado para la entrevista de informantes sobre el uso de plantas medicinales en la zona de Jenaro Herrera.

Nu. campo: Nu. Coll: Fam: Gen:

Especie: Nombre principal:

Otros nombres locales:

Fecha: Comunidad: Cuál terreno:

Informante: Edad: Sexo: Nacido:

1. Para cual enfermedad/motivo:

2. Parte de planta usada:

3. Cantidad de planta usada:

4. Mezcla con otros ingredientes:

5. Preparación de medicina vegetal:

6. Uso/cantidad/cuándo/duración:

7. Uso como parte de ritual/cuál/cómo:

8. Contraindicaciones:

9. Unico/el mejor/secundario:

10. Mercado/precios/dónde: 